\title{
Knowledge of cervical cancer risk factors among Palestinian women: a national cross-sectional study
}

\author{
Mohamedraed Elshami ${ }^{1,2^{*}{ }^{\dagger}}$, Mariam Thalji ${ }^{3 \dagger}$, Hanan Abukmail ${ }^{2,4 \dagger}$, Ibrahim Al-Slaibi ${ }^{5}$, Mohammed Alser ${ }^{2}$, \\ Afnan Radaydeh ${ }^{3}$, Alaa Alfuqaha ${ }^{6}$, Salma Khader ${ }^{3}$, Lana Khatib ${ }^{7}$, Nour Fannoun ${ }^{8}$, Bisan Ahmad ${ }^{4}$, Lina Kassab², \\ Hiba Khrishi ${ }^{9}$, Deniz Elhussaini ${ }^{10}$, Nour Abed $^{4}$, Aya Nammari $^{3}$, Tumodir Abdallah $^{3}$, Zaina Alqudwa ${ }^{10}$, \\ Shahd Idais ${ }^{3}$, Ghaid Tanbouz ${ }^{9}$, Ma'alem Hajajreh ${ }^{11}$, Hala Abu Selmiyh' ${ }^{4}$, Zakia Abo-Hajouj ${ }^{3}$, Haya Hebi ${ }^{3}$, \\ Manar Zamel ${ }^{7}$, Refqa Najeeb Skaik ${ }^{10}$, Lama Hammoud ${ }^{9}$, Saba Rjoub ${ }^{3}$, Hadeel Ayesh ${ }^{3}$, Toqa Rjoub ${ }^{3}$, \\ Rawan Zakout ${ }^{4}$, Amany Alser ${ }^{12}$, Nasser Abu-El-Noor ${ }^{13+}$ and Bettina Bottcher ${ }^{4 \dagger}$
}

\begin{abstract}
Background: High awareness of cervical cancer (CC) risk factors is important to decrease the morbidity and mortality associated with CC. This study aimed to assess the knowledge level of Palestinian women about CC risk factors and to determine the factors associated with good knowledge.

Methods: This was a national cross-sectional study. Adult women from hospitals, primary healthcare centers, and public spaces of 11 governorates in Palestine were recruited using a stratified convenience sampling. A translated-into-Arabic version of the validated CC awareness measure (CeCAM) was used to assess the knowledge about the 11 CC risk factors. For each correctly identified risk factor, the participant was given one point. The total score was calculated and was categorized into three categories: poor knowledge (0-3), fair knowledge (4-7), and good knowledge (8-11).
\end{abstract}

Results: A total of 7223 participants completed the Arabic CeCAM (response rate $=89.3 \%$ ) and 7058 questionnaires were included in the final analysis: 2655 from the Gaza Strip and 4403 from the West Bank and Jerusalem. Participants recruited from the Gaza Strip were younger, getting lower monthly incomes, and with less chronic diseases than participants recruited from the West Bank and Jerusalem. The most frequently identified risk factor was 'having a weakened immune system' $(n=5458,77.3 \%)$ followed by 'infection with a sexually transmitted infection' $(n=5388$, 76.3\%). The least identified risk factor was'having many children' $(n=1597,22.6 \%)$. Only 1670 women (23.7\%) had good knowledge of CC risk factors. Women living in the Gaza Strip were more likely than women living in the West Bank and Jerusalem to have good knowledge (25.2\% vs 22.7\%). Completing a secondary or diploma degree, being employed, and having a monthly income of $\geq 1450$ NIS (around \$450) were all associated with lower likelihood of

\footnotetext{
*Correspondence: mohamedraed.elshami@gmail.com

${ }^{\dagger}$ First co-author: Mohamedraed Elshami, Mariam Thalji and Hanan Abukmail

†'Senior co-author: Nasser Abu-El-Noor and Bettina Bottcher

${ }^{1}$ Harvard Medical School, 25 Shattuck Street, Boston, MA 02115, USA

Full list of author information is available at the end of the article
} original author(s) and the source, provide a link to the Creative Commons licence, and indicate if changes were made. The images or other third party material in this article are included in the article's Creative Commons licence, unless indicated otherwise in a credit line to the material. If material is not included in the article's Creative Commons licence and your intended use is not permitted by statutory regulation or exceeds the permitted use, you will need to obtain permission directly from the copyright holder. To view a copy of this licence, visit http://creativecommons.org/licenses/by/4.0/. The Creative Commons Public Domain Dedication waiver (http://creativeco mmons.org/publicdomain/zero/1.0/) applies to the data made available in this article, unless otherwise stated in a credit line to the data. 
having good knowledge of CC risk factors. Conversely, knowing someone with cancer was associated with higher likelihood of having good knowledge.

Conclusion: The overall awareness of CC risk factors was low. There is a substantial need to establish educational programs to promote Palestinian women's awareness of CC.

Keywords: Cervical cancer, Prevention, Early detection, Survival, Risk factor, Awareness, Knowledge, Early presentation, Palestine

\section{Introduction}

Cervical cancer $(\mathrm{CC})$ is the most commonly diagnosed gynecological cancer and one of the leading causes of cancer-related deaths in women worldwide [1, 2]. Globally, over 600,000 new cases and 300,000 deaths were estimated for CC in 2020 [1]. Half of these deaths occurred in countries of low and medium human development indices [1]. In Palestine, a lower-middle-income country, $\mathrm{CC}$ is the third most common gynecological cancer with an age-standardized incidence rate of 2.5 per 100,000 females [3-5]. CC in Palestine has a higher age-standardized mortality rate than other countries in the region [5]. This could be linked to the lack of an efficient screening program and diagnosis at later stages.

One of the main factors contributing to mortality of $\mathrm{CC}$ is diagnosis at a late stage $[6,7]$. This could be a result of several factors including low awareness of CC symptoms and risk factors as well as limited access to healthcare facilities particularly in low- and middle-income countries [8-12]. There are many key factors that can increase the risk of CC development. The most significant risk factor of $\mathrm{CC}$ is infection with human papillomavirus (HPV) [13-16]. HPV type 16 and 18 are high-risk sexually transmitted viruses and are responsible for more than $70 \%$ of CC cases [14-16]. Other behavioral and sexual factors that also may contribute to $\mathrm{CC}$ development include multiple sexual partners, early age of sexual intercourse, multiparty, sexual intercourse with an uncircumcised man, smoking and poor personal hygiene [17-22].

In Palestine, where there is no national screening program for CC, raising public awareness is crucial to reduce morbidity and mortality of CC. Good awareness of CC risk factors plays an essential role in early detection and thus improved prognosis $[6,9]$. Women who have good knowledge of CC risk factors are better able to recognize themselves as high-risk candidates to get the disease and therefore, they might seek medical advice earlier. Furthermore, women, who are aware to be at high-risk, are better equipped to adopt behaviors to reduce their probability of developing CC [23-25].

This national study aimed to: (1) assess Palestinian women's level of knowledge of CC risk factors, (2) identify the factors associated with a good knowledge level, and (3) compare the knowledge among women from the Gaza Strip vs. the West Bank and Jerusalem (WBJ).

\section{Materials and methods \\ Study design, setting and population}

A national cross-sectional study was conducted between July 2019 and March 2020 in Palestine. The Palestinian Ministry of Health $(\mathrm{MoH})$ hospitals and primary healthcare centers (PHCs) are the main entry sites for healthcare services in Palestine. These are distributed in two main geographical areas: (1) the Gaza Strip and (2) the WBJ. Therefore, governmental general hospitals with a bed capacity of more than 100 and PHCs with level four services (i.e., providing all primary healthcare services) were targeted to recruit participants into the study. Additionally, public spaces in the same governorates of hospitals and PHCs were involved, including markets, downtowns, mosques, churches, parks, malls, and restaurants.

In 2019, the estimated female population in Palestine was 2.45 million with about half of them in the reproductive age between 15 to 49 years [26]. Therefore, adult women aged 18 years or older were the target population and were invited to participate in the study. Potential participants were excluded if they had a citizenship other than Palestinian, were visiting the oncology departments, or were working or studying in a health-related field.

\section{Sampling methods}

The data collection process took place in 11 hospitals, 12 PHCs as well as 11 public spaces across Palestine. The hospitals had bed capacities of over 100, while the PHCs offered all services to the general Palestinian public. These sites were located across Palestine in different governorates covering a wide geographical area and were chosen for recruitment of participants by stratified convenience sampling.

\section{Questionnaire and data collection}

A translated-into-Arabic version of the validated Cervical Cancer Awareness Measure (CeCAM) was used [9]. The questionnaire consisted of two sections. The first section included socio-demographic questions. The second section comprised 11 questions based on a 5-point Likert 
scale $(1=$ strongly disagree, $5=$ strongly agree $)$ to assess the knowledge of CC risk factors.

The translation and adaptation of the questionnaire were performed based on World Health Organization recommendations [27]. The questionnaire was translated from English to Arabic by two healthcare professionals fluent in both languages and then back-translated into English by another two healthcare professionals who were also fluent in both languages. All healthcare professionals involved in this process had relevant clinical and research experience in gynecology, public health, and survey design.

A few items were adapted from the original CeCAM and were modified in the Arabic version to make them more culturally accepted in Palestine. 'Having a sexual partner who is not circumcised' was modified into 'having a husband who is not circumcised. Similarly, 'having a sexual partner with many previous partners' was modified into 'having a husband with many previous partners'. In addition, 'starting to have sex at a young age (before age 17)' was modified into 'being married at a young age (before age 17)'.

The Arabic CeCAM was modified for the purposes of this study. To minimize the possibility of participants answering questions at random, the original questions with yes/no/unknown responses were modified into 5-point Likert scale questions. Meanwhile, the participants' responses were then converted to correct/incorrect responses similar to what was done in previous studies [28-31].

A pilot study was conducted with 130 respondents to test the clarity of the items of the Arabic CeCAM version. These responses were not included in the final analysis. The Cronbach's Alpha showed that the questionnaire had an acceptable internal consistency $(\alpha=0.72)$.

Well-trained data collectors with a medical background conducted face-to-face interviews with the recruited participants for completion of the Arabic CeCAM. Data were collected utilizing the secure, user-friendly data collection tool 'Kobo Toolbox' that is accessed via smartphones [32].

\section{Statistical analysis}

Participant characteristics were summarized utilizing descriptive statistics. Continuous non-normally distributed variables were described using the median and interquartile range. Frequencies and percentages were utilized to summarize categorical variables. To reflect the age-associated risk of CC, age was categorized into three groups: $18-20$ years, $21-40$ years (at-risk group), and $\geq 41$ years [9]. A monthly income of 1450 NIS (about $\$ 450$ ) was the minimum wage in Palestine at the time of data collection [33]. Therefore, participants were categorized into two categories: $\geq 1450$ NIS and $<1450$ NIS. Baseline characteristics of participants from the WBJ vs. the Gaza Strip were compared using Pearson's Chi-square test if they were categorical or Kruskal-Wallis test if they were continuous.

For questions asking about $\mathrm{CC}$ risk factors, answering with 'strongly agree' and 'agree' was considered as a correct answer, whereas answering with 'strongly disagree', 'disagree', or 'not sure' was considered as an incorrect answer. Recognizing each $\mathrm{CC}$ risk factor was described using frequencies and percentages with comparisons utilizing Pearson's Chi-square test. This was followed by bivariable and multivariable logistic regression analyses. The model of the multivariable analysis adjusted for factors of socioeconomic status including age, educational level, occupation, monthly income, residency, and marital status. In addition, the model adjusted for other factors including having a chronic disease, knowing someone with cancer, and site of data collection. The model was pre-specified based on previous studies [9, 34-36]. Results of all bivariable logistic regression analyses were provided in Additional file 1.

To evaluate the knowledge level of CC risk factors, a scoring system was used. Similar scoring systems had been adopted in previous studies [24, 28]. For each correctly identified risk factor, the participant was given one point. The total score was then calculated (ranging from 0 to 11) and was categorized into three categories: poor knowledge (0-3), fair knowledge (4-7), and good knowledge (8-11). The knowledge level between the participants from the Gaza Strip and the WBJ was compared using Pearson's Chi-square test. Bivariable and multivariable logistic regression analyses were used to test the association between participants' characteristics and having a good knowledge level.

Complete case analysis was used to handle missing data (i.e., cases with incomplete data were excluded from the analysis; a total of 135 cases). The missing data were completely random and unrelated to the study variables. Data were analyzed using Stata software version 16.0 (StataCorp, College Station, Texas, United States).

\section{Results}

\section{Participant characteristics}

A total of 7223 participants, out of 8086 approached, completed the questionnaire (response rate $=89.3 \%$ ). The final analysis included 7058 questionnaires (30 did not meet inclusion criteria and 135 had missing values); 4403 from the WBJ and 2655 from the Gaza Strip.

The median age [interquartile range] for all participants was 32.0 years [24.0, 42.0] (Table 1). Participants recruited from the Gaza Strip were younger, getting 
Table 1 Characteristics of study participants

\begin{tabular}{|c|c|c|c|}
\hline Characteristic & Total $(n=7058)$ & Gaza strip $(n=2655)$ & WBJ $(n=4403)$ \\
\hline Age, median [IQR] & $32[24,42]$ & $30[24,39]$ & $33[24,44]$ \\
\hline \multicolumn{4}{|l|}{ Age group, n (\%) } \\
\hline $18-20$ & $756(10.7)$ & $249(9.4)$ & $507(11.5)$ \\
\hline $21-40$ & $4331(61.4)$ & $1809(68.1)$ & $2522(57.3)$ \\
\hline 41 or older & $1971(27.9)$ & $597(22.5)$ & $1374(31.2)$ \\
\hline \multicolumn{4}{|l|}{ Educational level, n (\%) } \\
\hline Illiterate & $127(1.8)$ & $37(1.4)$ & $90(2.0)$ \\
\hline Primary & $409(5.8)$ & $127(4.8)$ & $282(6.4)$ \\
\hline Preparatory & $1064(15.1)$ & $378(14.2)$ & $686(15.6)$ \\
\hline Secondary & $2293(32.5)$ & $955(36.0)$ & $1338(30.4)$ \\
\hline Diploma & $766(10.9)$ & $303(11.4)$ & $463(10.5)$ \\
\hline Bachelor & $2261(32.0)$ & $817(30.8)$ & $1444(32.8)$ \\
\hline Postgraduate & $138(1.9)$ & $38(1.4)$ & $100(2.3)$ \\
\hline \multicolumn{4}{|l|}{ Occupation, n (\%) } \\
\hline Housewife & $4647(65.8)$ & $2008(75.6)$ & $2639(59.9)$ \\
\hline Employed & $1476(20.9)$ & $348(13.1)$ & $1128(25.6)$ \\
\hline Retired & $69(1.0)$ & $11(0.4)$ & $58(1.3)$ \\
\hline Student & $866(12.3)$ & $288(10.9)$ & $578(13.2)$ \\
\hline Monthly income $\geq 1450$ NIS, n (\%) & $4666(66.1)$ & $693(26.1)$ & $3973(90.2)$ \\
\hline Having a chronic disease, n (\%) & $1397(19.8)$ & $417(15.7)$ & $980(22.3)$ \\
\hline Knowing someone with cancer, $\mathrm{n}(\%)$ & $4083(57.9)$ & $1483(55.9)$ & $2600(59.1)$ \\
\hline \multicolumn{4}{|l|}{ Marital status, n (\%) } \\
\hline Single & $1657(23.4)$ & $527(19.8)$ & $1130(25.6)$ \\
\hline Married & $5058(71.7)$ & $2025(76.3)$ & $3033(68.9)$ \\
\hline Divorced & $154(2.2)$ & $45(1.7)$ & $109(2.5)$ \\
\hline Widowed & $189(2.7)$ & $58(2.2)$ & $131(3.0)$ \\
\hline \multicolumn{4}{|l|}{ Site of data collection } \\
\hline Public spaces, n (\%) & $2695(38.2)$ & $863(32.5)$ & $1832(41.7)$ \\
\hline Hospitals, n (\%) & $1890(26.8)$ & $642(24.2)$ & $1248(28.3)$ \\
\hline Primary healthcare centers, n (\%) & $2473(35.0)$ & $1150(43.3)$ & $1323(30.0)$ \\
\hline
\end{tabular}

n, number of participants; IQR, interquartile range; WBJ, West Bank and Jerusalem

Table 2 Knowledge level among study participants

\begin{tabular}{llccc}
\hline Level & $\begin{array}{l}\text { Total } \\
\mathbf{n}(\%)\end{array}$ & $\begin{array}{l}\text { Gaza strip } \\
\mathbf{n}(\%)\end{array}$ & $\begin{array}{l}\text { WBJ } \\
\mathbf{n}(\%)\end{array}$ & $\boldsymbol{p}$ value \\
\hline Poor & $1140(16.1)$ & $374(14.1)$ & $766(17.4)$ & $<0.001$ \\
Fair & $4248(60.2)$ & $1611(60.7)$ & $2637(59.9)$ & \\
Good & $1670(23.7)$ & $670(25.2)$ & $1000(22.7)$ & \\
\hline
\end{tabular}

$\mathrm{n}$, number of participants; WBJ, West Bank and Jerusalem

lower monthly income, and with less chronic diseases than participants recruited from the WBJ.

\section{Good knowledge and its associated factors}

Only 1670 women (23.7\%) had a good knowledge of CC risk factors (Table 2). Women living in the Gaza Strip were more likely than women living in the $\mathrm{WBJ}$ to have good knowledge (25.2\% vs $22.7 \%$ ).

On the multivariable analysis, completing secondary or diploma degree, being employed, and having a monthly income of $\geq 1450$ NIS were all associated with a decrease in the odds of having good knowledge of CC risk factors (Table 3). On the other hand, knowing someone with cancer was associated with an increase in the odds of having good knowledge.

\section{Recognition of CC risk factors in the Gaza strip versus the WBJ}

Among all participants, the most frequently recognized risk factor was 'having a weakened immune system' $(\mathrm{n}=5458,77.3 \%)$ followed by 'infection with a sexually transmitted infection (STI)' $(\mathrm{n}=5388,76.3 \%)$ (Table 4). These risk factors were also the most recognized factors 
Table 3 Association between having a good knowledge and sociodemographic factors

\begin{tabular}{|c|c|c|c|c|c|}
\hline \multirow[t]{2}{*}{ Characteristic } & \multicolumn{5}{|c|}{ Good knowledge } \\
\hline & n (\%) & COR $(95 \% \mathrm{Cl})$ & $p$ value & $\operatorname{AOR}(95 \% \mathrm{Cl})^{\mathrm{a}}$ & $p$ value \\
\hline \multicolumn{6}{|l|}{ Age group } \\
\hline $18-20$ & $157(9.4)$ & Ref & Ref & Ref & Ref \\
\hline $21-40$ & $1016(60.8)$ & $1.17(0.97-1.41)$ & 0.11 & $1.17(0.92-1.48)$ & 0.20 \\
\hline 41 or older & $497(29.8)$ & $1.29(1.05-1.58)$ & 0.015 & $129(0.98-1.69)$ & 0.07 \\
\hline \multicolumn{6}{|l|}{ Educational level } \\
\hline Illiterate & $40(2.4)$ & Ref & Ref & Ref & Ref \\
\hline Primary & $112(6.7)$ & $0.82(0.53-1.26)$ & 0.37 & $0.75(0.48-1.17)$ & 0.21 \\
\hline Preparatory & $260(15.6)$ & $0.70(0.47-1.05)$ & 0.08 & $0.67(0.44-1.00)$ & 0.051 \\
\hline Secondary & $513(30.7)$ & $0.63(0.43-0.92)$ & 0.018 & $0.63(0.42-0.94)$ & 0.023 \\
\hline Diploma & $162(9.7)$ & $0.58(0.39-0.88)$ & 0.010 & $0.63(0.41-0.97)$ & 0.035 \\
\hline Bachelor & $540(32.3)$ & $0.68(0.46-1.00)$ & 0.053 & $0.76(0.50-1.14)$ & 0.19 \\
\hline Postgraduate & $43(2.6)$ & $0.98(0.59-1.66)$ & 0.95 & $1.17(0.67-2.04)$ & 0.57 \\
\hline \multicolumn{6}{|l|}{ Occupation } \\
\hline Housewife & $1144(68.5)$ & Ref & Ref & Ref & Ref \\
\hline Employed & $316(18.9)$ & $0.83(0.72-0.96)$ & 0.012 & $0.81(0.68-0.96)$ & 0.016 \\
\hline Retired & $13(0.8)$ & $0.71(0.39-1.30)$ & 0.27 & $0.76(0.40-1.43)$ & 0.39 \\
\hline Student & $197(11.8)$ & $0.90(0.76-1.07)$ & 0.24 & $1.05(0.82-1.35)$ & 0.71 \\
\hline \multicolumn{6}{|l|}{ Monthly income } \\
\hline$<1450$ NIS & $619(37.1)$ & Ref & Ref & Ref & Ref \\
\hline$\geq 1450 \mathrm{NIS}$ & $1051(62.9)$ & $0.83(0.74-0.93)$ & 0.002 & $0.85(0.72-0.99)$ & 0.038 \\
\hline \multicolumn{6}{|l|}{ Marital status } \\
\hline Single & $362(21.7)$ & Ref & Ref & Ref & Ref \\
\hline Married & $1226(73.4)$ & $1.14(1.00-1.31)$ & 0.047 & $1.06(0.89-1.26)$ & 0.55 \\
\hline Divorced & $39(2.3)$ & $1.21(0.83-1.78)$ & 0.32 & $1.17(0.79-1.74)$ & 0.44 \\
\hline Widowed & $43(2.6)$ & $1.05(0.74-1.51)$ & 0.78 & $0.85(0.57-1.26)$ & 0.41 \\
\hline \multicolumn{6}{|l|}{ Residency } \\
\hline Gaza strip & $670(40.1)$ & Ref & Ref & Ref & Ref \\
\hline WBJ & $1000(59.9)$ & $0.87(0.78-0.97)$ & 0.016 & $0.97(0.83-1.13)$ & 0.66 \\
\hline \multicolumn{6}{|l|}{ Having a chronic disease } \\
\hline No & $1314(78.7)$ & Ref & Ref & Ref & Ref \\
\hline Yes & $356(21.3)$ & $1.13(0.99--1.30)$ & 0.07 & $1.05(0.90-1.22)$ & 0.58 \\
\hline \multicolumn{6}{|l|}{ Knowing someone with cancer } \\
\hline No & $611(36.6)$ & Ref & Ref & Ref & Ref \\
\hline Yes & $1059(63.4)$ & $1.34(1.20-1.51)$ & $<0.001$ & $1.34(1.19-1.50)$ & $<0.001$ \\
\hline \multicolumn{6}{|l|}{ Site of data collection } \\
\hline Public spaces & $636(38.1)$ & Ref & Ref & Ref & Ref \\
\hline Hospitals & $442(26.5)$ & $0.99(0.86-1.14)$ & 0.87 & $0.94(0.81-1.10)$ & 0.45 \\
\hline Primary healthcare centers & $592(35.4)$ & $1.02(0.90-1.16)$ & 0.78 & $0.96(0.84-1.10)$ & 0.58 \\
\hline
\end{tabular}

COR, crude odds ratio; $\mathrm{AOR}$, adjusted odds ratio; $\mathrm{Cl}$, confidence interval; WBJ, West Bank and Jerusalem

${ }^{a}$ Adjusted for age-group, educational level, occupation, monthly income, marital status, residency, having a chronic disease, knowing someone with cancer, and site of data collection

in both the Gaza Strip and WBJ. The least recognized risk factors were 'having many children' $(\mathrm{n}=1597,22.6 \%)$ and 'being married at a young age' $(\mathrm{n}=2197,31.1 \%)$.

The Chi-square test showed that participants from the Gaza Strip had a higher likelihood than participants from the WBJ to recognize 'having a weakened immune system,' 'infection with a sexually transmitted infection (STI)', 'infection with HPV', 'not going for regular Pap smears', and 'having uncircumcised husband'. On the other hand, participants from the WBJ were more likely to recognize 'having a relative with $C C$ ', 'having a husband with many previous partners', 'being married at a 
Table 4 Recognition of cervical cancer risk factors

\begin{tabular}{|c|c|c|c|c|}
\hline Risk factor & $\begin{array}{l}\text { Total }(n=7058) \\
n(\%)\end{array}$ & $\begin{array}{l}\text { Gaza strip }(\mathrm{n}=2655) \\
\mathrm{n}(\%)\end{array}$ & $\begin{array}{l}\text { WBJ }(n=4403) \\
n(\%)\end{array}$ & $p$ value \\
\hline Having a weakened immune system & $5458(77.3)$ & $2139(80.6)$ & $3319(75.4)$ & $<0.001$ \\
\hline Infection with a sexually transmitted infection & $5388(76.3)$ & $2132(80.3)$ & $3256(73.9)$ & $<0.001$ \\
\hline Infection with human papillomavirus (HPV) & $4693(66.5)$ & $1977(74.5)$ & $2716(61.7)$ & $<0.001$ \\
\hline Having a relative with cervical cancer & $4250(60.2)$ & $1538(57.9)$ & $2712(61.6)$ & 0.002 \\
\hline Long term use of the contraceptive pill & $4236(60.0)$ & $1620(61.0)$ & $2616(59.4)$ & 0.18 \\
\hline Smoking any cigarettes at all & $4167(59.0)$ & $1600(60.3)$ & $2567(58.3)$ & 0.10 \\
\hline Not going for regular smear (Pap) tests & $3543(50.2)$ & $1507(56.8)$ & $2036(46.2)$ & $<0.001$ \\
\hline Having a husband who is not circumcised & $2818(39.9)$ & $1127(42.4)$ & $1691(38.4)$ & $<0.001$ \\
\hline Having a husband with many previous partners & $2562(36.3)$ & $842(31.7)$ & $1720(39.1)$ & $<0.001$ \\
\hline Being married at a young age (before age 17) & $2197(31.1)$ & $779(29.3)$ & $1418(32.2)$ & 0.012 \\
\hline Having many children (five or more) & $1597(22.6)$ & $559(21.1)$ & $1038(23.6)$ & 0.014 \\
\hline
\end{tabular}

n, number of participants; WBJ, West Bank and Jerusalem

young age', and 'having many children' as risk factors for CC.

\section{Association between recognizing $\mathrm{CC}$ risk factors and socioeconomic status}

On the multivariable analysis, women with age-related risk of CC (aged 21-40 years) were less likely than younger women (aged 18-20 years) to recognize infection with an STI' (OR $=0.71,95 \%$ CI 0.56-0.91), 'infection with HPV' (OR=0.76, 95\% CI 0.61-0.96), and 'not going to regular Pap smears' $(\mathrm{OR}=0.75,95 \% \mathrm{CI} 0.61$ 0.91 ) as risk factors for CC (Tables 5, 6).

Participants with a bachelor degree had a higher likelihood than illiterate participants to identify 'having a weakened immune system' $(\mathrm{OR}=2.92,95 \% \mathrm{CI}$ 1.94-4.39), 'infection with an STI' $(\mathrm{OR}=2.40,95 \% \mathrm{CI}$ $1.60-3.60)$, and 'infection with HPV' (OR $=1.69,95 \%$ CI 1.14-2.50) as risk factors for CC. However, participants who had a bachelor degree were less likely to identify 'having a husband with many previous partners' $(\mathrm{OR}=0.56,95 \% \mathrm{CI} 0.36-0.83)$ as a CC risk factor.

Married women were more likely than single women to recognize 7 out of $11 \mathrm{CC}$ risk factors. Moreover, participants with a monthly income of $\geq 1450$ NIS had a higher likelihood than participants with a lower monthly income to recognize having a weakened immune system' $(\mathrm{OR}=1.22,95 \% \mathrm{CI} 1.03-1.44)$ and 'infection with an $\mathrm{STI}^{\prime}(\mathrm{OR}=1.19,95 \% \mathrm{CI} 1.01-1.40)$ as risk factors for CC. Nonetheless, participants earning $\geq 1450$ NIS had a lower likelihood to recognize other CC risk factors including 'not going to regular Pap smears', 'having a husband with many previous partners', 'being married at a young age', and 'having many children'. In addition, employed women were less likely than unemployed or housewives to recognize 'infection with an STI' $(\mathrm{OR}=0.72$, 95\%
CI 0.61-0.85), 'infection with HPV' $(\mathrm{OR}=0.80,95 \%$ CI 0.69-0.93), and 'not going to regular Pap smears' $(\mathrm{OR}=0.87,95 \%$ CI $0.75-0.99)$.

\section{Association between recognizing CC risk factors and other participants' characteristics}

Women who knew someone with cancer were more likely than women who did not to identify all CC risk factors except 'having a husband with many previous partners', 'being married at a young age', and 'having many children' for which no differences were found.

Participants visiting hospitals were less likely than participants visiting public spaces to identify 'infection with an STI', 'infection with HPV', 'having a relative with CC, and 'long term use of the contraceptive pill. However, hospital visitors were more likely to identify 'having a husband with many previous partners' $(\mathrm{OR}=1.18$, 95\% CI 1.03-1.34).

Participants visiting PHCs were less likely than participants visiting public spaces to identify 'infection with an STI', 'infection with HPV', 'having a husband who is not circumcised, 'having a husband with many previous partners', and 'being married at a young age' as risk factor for CC. However, visitors to PHCs were more likely to identify other CC risk factors including 'having a weakened immune system,' 'having a relative with CC', 'smoking any cigarettes at all', and 'not going for regular Pap smears'.

\section{Discussion}

The overall awareness of CC risk factors in this study was low with only $23.7 \%$ of the participants having good knowledge. Knowing someone with cancer was associated with an increase in the odds of having good knowledge. Participants from the Gaza Strip demonstrated better knowledge than participants from the 
Table 5 Multivariable logistic regression analyzing the association between the recognition of the most identified risk factors and sociodemographic factors

\begin{tabular}{|c|c|c|c|c|c|c|c|c|c|c|c|}
\hline \multirow[t]{2}{*}{ Characteristic } & \multicolumn{4}{|c|}{$\begin{array}{l}\text { Having a weakened immune system } \\
(n=5458)\end{array}$} & \multicolumn{4}{|c|}{$\begin{array}{l}\text { Infection with a sexually transmitted } \\
\text { infection }(n=5388)\end{array}$} & \multicolumn{3}{|c|}{ Infection with HPV ( $n=4693)$} \\
\hline & \multirow[t]{2}{*}{ n (\%) } & \multicolumn{2}{|c|}{ AOR $(95 \% \mathrm{Cl})^{\mathrm{a}}$} & $p$ value & \multicolumn{2}{|c|}{ n (\%) } & AOR $(95 \% \mathrm{Cl})^{\mathrm{a}}$ & $p$ value & n (\%) & $\operatorname{AOR}(95 \% \mathrm{Cl})^{\mathrm{a}}$ & $p$ value \\
\hline \multicolumn{11}{|l|}{ Age group } & \\
\hline $18-20$ & $560(10.3)$ & \multicolumn{2}{|l|}{ Ref } & Ref & \multicolumn{2}{|c|}{$607(11.3)$} & Ref & Ref & $574(12.2)$ & Ref & Ref \\
\hline $21-40$ & 3355 (61.5) & $0.97(C$ & .77-1.22) & 0.81 & 3329 & 61.8) & $0.71(0.56-0.91)$ & 0.006 & $2878(61.3)$ & $0.76(0.61-0.96)$ & 0.018 \\
\hline 41 or older & $1543(28.3)$ & $1.16(C$ & 89-1.51) & 0.29 & 1452 & 26.9) & $0.74(0.56-0.97)$ & 0.029 & $1241(26.4)$ & $0.81(0.63-1.04)$ & 0.10 \\
\hline Educational level & & & & & & & & & & & \\
\hline Illiterate & $78(1.4)$ & Ref & & Ref & & 1.5) & Ref & Ref & $73(1.6)$ & Ref & Ref \\
\hline Primary & $294(5.4)$ & $1.42(C$ & $.93-2.19)$ & 0.11 & 265 & 4.9) & $1.00(0.66-1.53)$ & 0.99 & $241(5.1)$ & $1.08(0.71-1.63)$ & 0.73 \\
\hline Preparatory & 828 (15.2) & 2.01 & $.34-3.00)$ & 0.001 & 801 & 14.9) & $1.62(1.09-2.41)$ & 0.018 & $664(14.1)$ & $1.22(0.83-1.80)$ & 0.31 \\
\hline Secondary & 1805 (33.1) & 2.44( & .64-3.62) & $<0.001$ & 1787 & 33.2) & $1.90(1.28-2.81)$ & 0.001 & $1513(32.2)$ & $1.29(0.88-1.89)$ & 0.18 \\
\hline Diploma & 559 (10.2) & 2.08 & $.37-3.17)$ & 0.001 & 563 & 10.4) & $1.84(1.21-2.81)$ & 0.004 & $511(10.9)$ & $1.52(1.01-2.28)$ & 0.043 \\
\hline Bachelor & 1777 (32.6) & 2.92( & $.94-4.39)$ & $<0.001$ & 1778 & 33.0) & $2.40(1.60-3.60)$ & $<0.001$ & 1589 (33.9) & $1.69(1.14-2.50)$ & 0.009 \\
\hline Postgraduate & $117(2.1)$ & 4.63 & $.48-8.65)$ & $<0.001$ & 114 & 2.1) & $3.64(1.99-6.66)$ & $<0.001$ & $102(2.2)$ & $2.47(1.42-4.28)$ & 0.001 \\
\hline Occupation & & & & & & & & & & & \\
\hline Housewife & $3653(66.9)$ & Ref & & Ref & 3591 & 66.6) & Ref & Ref & $3043(64.8)$ & Ref & Ref \\
\hline Employed & $1122(20.6)$ & $0.92(C$ & $.78-1.09)$ & 0.35 & 1083 & 20.1) & $0.72(0.61-0.85)$ & $<0.001$ & $945(20.1)$ & $0.80(0.69-0.93)$ & 0.004 \\
\hline Retired & $39(0.7)$ & $0.38(C$ & $.22-0.65)$ & $<0.001$ & & 0.6) & $0.28(0.17-0.47)$ & $<0.001$ & $34(0.7)$ & $0.53(0.32-0.88)$ & 0.014 \\
\hline Student & $644(11.8)$ & 1.09 (C & $.85-1.40)$ & 0.49 & 682 & 12.7) & $0.89(0.69-1.15)$ & 0.38 & $671(14.3)$ & $1.31(1.03-1.67)$ & 0.027 \\
\hline Monthly income & & & & & & & & & & & \\
\hline$<1450$ NIS & $1867(34.2)$ & Ref & & Ref & 1851 & 34.4) & Ref & Ref & $1700(36.2)$ & Ref & Ref \\
\hline$\geq 1450 \mathrm{NIS}$ & 3591 (65.8) & 1.22( & $.03-1.44)$ & 0.020 & 3537 & 65.6) & $1.19(1.01-1.40)$ & 0.041 & $2993(63.8)$ & $0.97(0.84-1.13)$ & 0.74 \\
\hline Residency & & & & & & & & & & & \\
\hline Gaza Strip & 2139 (39.2) & Ref & & Ref & 2132 & 39.6) & Ref & Ref & $1977(42.1)$ & Ref & Ref \\
\hline WBJ & $3319(60.8)$ & 0.70 & $.59-0.82)$ & $<0.001$ & 3256 & 60.4) & $0.67(0.57-0.79)$ & $<0.001$ & $2716(57.9)$ & $0.53(0.46-0.61)$ & $<0.001$ \\
\hline Having a chronic disease & & & & & & & & & & & \\
\hline No & $4358(79.8)$ & Ref & & Ref & 4344 & 80.6) & Ref & Ref & $3815(81.3)$ & Ref & Ref \\
\hline Yes & $1100(20.2)$ & $1.17(($ & $.99-1.38)$ & 0.07 & 1044 & 19.4) & $1.12(0.96-1.31)$ & 0.15 & $878(18.7)$ & $0.99(0.86-1.14)$ & 0.88 \\
\hline Knowing someone with cance & & & & & & & & & & & \\
\hline No & $2180(39.9)$ & Ref & & Ref & 2206 & 40.9) & Ref & Ref & $1891(40.3)$ & Ref & Ref \\
\hline Yes & $3278(60.1)$ & $1.41(1$ & 25-1.58) & $<0.001$ & 3182 & 59.1) & $1.15(1.03-1.29)$ & 0.015 & $2802(59.7)$ & $1.23(1.11-1.37)$ & $<0.001$ \\
\hline Marital status & & & & & & & & & & & \\
\hline Single & $1183(21.7)$ & Ref & & Ref & 1229 & 22.8) & Ref & Ref & $1168(24.9)$ & Ref & Ref \\
\hline Married & $4021(73.7)$ & 1.55( & $.30-1.83)$ & $<0.001$ & 3918 & 72.7) & $1.38(1.17-1.64)$ & $<0.001$ & $3306(70.4)$ & $1.08(0.92-1.26)$ & 0.36 \\
\hline Divorced & $112(2.1)$ & $1.18(($ & $.80-1.74)$ & 0.40 & 114 & 2.1) & $1.25(0.84-1.85)$ & 0.27 & $102(2.2)$ & $1.11(0.77-1.60)$ & 0.59 \\
\hline Widowed & $142(2.6)$ & 1.51( & $.02-2.23)$ & 0.039 & 127 & 2.4) & $1.05(0.73-1.51)$ & 0.79 & $117(2.5)$ & $1.06(0.75-1.50)$ & 0.75 \\
\hline Site of data collection & & & & & & & & & & & \\
\hline Public spaces & 2015 (36.9) & Ref & & Ref & 2113 & (39.2) & Ref & Ref & $1966(41.9)$ & Ref & Ref \\
\hline Hospitals & $1423(26.1)$ & 1.04( & $.89-1.20)$ & 0.64 & 1362 & 25.3) & $0.72(0.62-0.84)$ & $<0.001$ & $1216(25.9)$ & $0.73(0.64-0.84)$ & $<0.001$ \\
\hline Primary healthcare centers & $2020(37.0)$ & 1.41( & $.22-1.63)$ & $<0.001$ & 1913 & 35.5) & $0.86(0.75-0.99)$ & 0.040 & $1511(32.2)$ & $0.56(0.49-0.64)$ & $<0.001$ \\
\hline $\begin{array}{l}\text { Characteristic Having a re } \\
\qquad(\mathrm{n}=4250)\end{array}$ & elative with ce & ervical & cancer & $\begin{array}{l}\text { Long } t \\
(\mathrm{n}=42 \\
\end{array}$ & $\begin{array}{l}\text { erm u } \\
\text { 236) }\end{array}$ & of th & e contraceptive & & $\begin{array}{l}\text { Smoking any } \\
(n=4167)\end{array}$ & cigarettes at all & \\
\hline n (\%) & AOR $(95 \%$ & $\% \mathrm{Cl})^{\mathrm{a}}$ & $p$ value & n (\%) & & AOR & $(95 \% \mathrm{Cl})^{\mathrm{a}}$ & value & $n(\%)$ & AOR $(95 \% \mathrm{Cl})^{\mathrm{a}}$ & $p$ value \\
\hline Age group & & & & & & & & & & & \\
\hline $418(9.8)$ & Ref & & Ref & $446(1$ & 0.5) & Ref & R & ef & $405(9.7)$ & Ref & Ref \\
\hline $2629(61.9)$ & $1.05(0.86$ & $-1.29)$ & 0.61 & $2588(6$ & 51.1) & 0.96 & $(0.79-1.17)$ & .71 & $2566(61.6)$ & $1.02(0.83-1.24)$ & 0.88 \\
\hline 41 or older & $1.01(0.81$ & $-1.28)$ & 0.90 & 1202 & 28.4) & 1.02 & $(0.81-1.28)$ & 90 & 1196 (28.7) & $0.96(0.76-1.21)$ & 0.72 \\
\hline
\end{tabular}


Table 5 (continued)

\begin{tabular}{|c|c|c|c|c|c|c|c|c|c|}
\hline \multirow[t]{2}{*}{ Characteristic } & \multicolumn{3}{|c|}{$\begin{array}{l}\text { Having a relative with cervical cancer } \\
(n=4250)\end{array}$} & \multicolumn{3}{|c|}{$\begin{array}{l}\text { Long term use of the contraceptive pill } \\
(n=4236)\end{array}$} & \multicolumn{3}{|c|}{$\begin{array}{l}\text { Smoking any cigarettes at all } \\
(n=4167)\end{array}$} \\
\hline & n (\%) & $\operatorname{AOR}(95 \% \mathrm{CI})^{\mathrm{a}}$ & $p$ value & n (\%) & AOR $(95 \% \mathrm{CI})^{\mathrm{a}}$ & $p$ value & n (\%) & AOR $(95 \% \mathrm{CI})^{\mathrm{a}}$ & $p$ value \\
\hline \multicolumn{10}{|l|}{ Educational level } \\
\hline Illiterate & $78(1.8)$ & Ref & Ref & $77(1.8)$ & Ref & Ref & $82(2.0)$ & Ref & Ref \\
\hline Primary & $264(6.2)$ & $1.02(0.67-1.55)$ & 0.93 & $240(5.7)$ & $0.89(0.59-1.34)$ & 0.56 & $258(6.2)$ & $0.89(0.59-1.36)$ & 0.61 \\
\hline Preparatory & $685(16.1)$ & $0.96(0.65-1.42)$ & 0.85 & $631(14.9)$ & $0.91(0.62-1.34)$ & 0.63 & 701 (16.8) & $0.99(0.67-1.48)$ & 0.99 \\
\hline Secondary & 1387 (32.6) & $0.86(0.58-1.25)$ & 0.42 & $1346(31.8)$ & $0.92(0.63-1.35)$ & 0.67 & $1371(32.9)$ & $0.83(0.56-1.22)$ & 0.34 \\
\hline Diploma & 414 (9.7) & $0.72(0.48-1.08)$ & 0.12 & $428(10.1)$ & $0.87(0.58-1.29)$ & 0.49 & $429(10.3)$ & $0.79(0.53-1.19)$ & 0.26 \\
\hline Bachelor & 1338 (31.5) & $0.88(0.59-1.30)$ & 0.52 & $1417(33.5)$ & $1.13(0.76-1.66)$ & 0.55 & 1245 (29.9) & $0.78(0.52-1.16)$ & 0.22 \\
\hline Postgraduate & $84(2.0)$ & $0.91(0.54-1.55)$ & 0.74 & $97(2.3)$ & $1.56(0.91-2.66)$ & 0.11 & $81(1.9)$ & $0.94(0.55-1.58)$ & 0.81 \\
\hline \multicolumn{10}{|l|}{ Occupation } \\
\hline Housewife & $2900(68.2)$ & Ref & Ref & 2776 (65.5) & Ref & Ref & 2884 (69.2) & Ref & Ref \\
\hline Employed & $854(20.1)$ & $0.91(0.79-1.05)$ & 0.21 & $907(21.4)$ & $1.01(0.88-1.17)$ & 0.85 & 796 (19.1) & $0.93(0.80-1.07)$ & 0.29 \\
\hline Retired & $29(0.7)$ & $0.63(0.38-1.06)$ & 0.08 & $33(0.8)$ & $0.66(0.40-1.10)$ & 0.11 & $33(0.8)$ & $0.79(0.47-1.31)$ & 0.35 \\
\hline Student & $467(11.0)$ & $0.88(0.71-1.09)$ & 0.23 & $520(12.3)$ & $1.08(0.87-1.34)$ & 0.47 & $454(10.9)$ & $1.08(0.88-1.34)$ & 0.46 \\
\hline \multicolumn{10}{|l|}{ Monthly income } \\
\hline$<1450$ NIS & $1394(32.8)$ & Ref & Ref & $1413(33.4)$ & Ref & Ref & $1476(35.4)$ & Ref & Ref \\
\hline$\geq 1450 \mathrm{NIS}$ & $2856(67.2)$ & $1.13(0.98-1.30)$ & 0.09 & $2823(66.6)$ & $1.13(0.98-1.30)$ & 0.09 & $2691(64.6)$ & $0.93(0.81-1.08)$ & 0.34 \\
\hline \multicolumn{10}{|l|}{ Residency } \\
\hline Gaza Strip & $1538(36.2)$ & Ref & Ref & $1620(38.2)$ & Ref & Ref & $1600(38.4)$ & Ref & Ref \\
\hline WBJ & $2712(63.8)$ & $1.19(1.04-1.36)$ & 0.011 & $2616(61.8)$ & $0.85(0.74-0.98)$ & 0.021 & 2567 (61.6) & $1.04(0.91-1.19)$ & 0.59 \\
\hline \multicolumn{10}{|c|}{ Having a chronic disease } \\
\hline No & $3408(80.2)$ & Ref & Ref & 3367 (79.5) & Ref & Ref & 3314 (79.5) & Ref & Ref \\
\hline Yes & $842(19.8)$ & $0.91(0.79-1.04)$ & 0.18 & $869(20.5)$ & $1.14(0.99-1.30)$ & 0.07 & $853(20.5)$ & $0.98(0.86-1.13)$ & 0.81 \\
\hline \multicolumn{10}{|c|}{ Knowing someone with cancer } \\
\hline No & $1671(39.3)$ & Ref & Ref & $1666(39.3)$ & Ref & Ref & $1684(40.4)$ & Ref & Ref \\
\hline Yes & $2579(60.7)$ & $1.33(1.20-1.47)$ & $<0.001$ & $2570(60.7)$ & $1.28(1.16-1.42)$ & $<0.001$ & $2483(59.6)$ & $1.21(1.10-1.34)$ & $<0.001$ \\
\hline \multicolumn{10}{|l|}{ Marital status } \\
\hline Single & $895(21.1)$ & Ref & Ref & $954(22.5)$ & Ref & Ref & $829(19.9)$ & Ref & Ref \\
\hline Married & $3165(74.5)$ & $1.22(1.05-1.42)$ & 0.009 & $3073(72.5)$ & $1.26(1.08-1.46)$ & 0.003 & $3121(74.9)$ & $1.41(1.21-1.63)$ & $<0.001$ \\
\hline Divorced & $80(1.9)$ & $0.88(0.62-1.24)$ & 0.45 & $96(2.3)$ & $1.33(0.94-1.90)$ & 0.11 & $90(2.2)$ & $1.37(0.97-1.94)$ & 0.07 \\
\hline Widowed & $110(2.6)$ & $1.06(0.76-1.49)$ & 0.72 & $113(2.7)$ & $1.19(0.85-1.67)$ & 0.31 & $127(3.0)$ & $1.81(1.28-2.57)$ & 0.001 \\
\hline \multicolumn{10}{|c|}{ Site of data collection } \\
\hline Public spaces & $1531(36.0)$ & Ref & Ref & $1666(39.3)$ & Ref & Ref & 1432(34.4) & Ref & Ref \\
\hline Hospitals & $1050(24.7)$ & $0.87(0.77-0.99)$ & 0.037 & $1091(25.8)$ & $0.85(0.75-0.97)$ & 0.016 & $1095(26.3)$ & $1.11(0.98-1.26)$ & 0.10 \\
\hline $\begin{array}{l}\text { Primary health- } \\
\text { care centers }\end{array}$ & 1669 (39.3) & $1.52(1.34-1.72)$ & $<0.001$ & 1479 (34.9) & $0.92(0.82-1.04)$ & 0.18 & 1640 (39.4) & $1.58(1.40-1.79)$ & $<0.001$ \\
\hline
\end{tabular}

n, number of participants; AOR, adjusted odds ratio; Cl, confidence interval; WBJ, West Bank and Jerusalem; HPV, human papillomavirus

${ }^{\text {a }}$ Adjusted for age-group, educational level, occupation, monthly income, marital status, residency, having a chronic disease, knowing someone with cancer, and site of data collection

WBJ. 'Having a weakened immune system' was the most reported CC risk factor followed by infection with an STI. The least reported risk factors were 'having many children' and 'being married at a young age'.

High awareness of CC risk factors could play an essential role in the prevention and early detection of CC [37, 38]. This study evaluated the Palestinian women's level of knowledge of CC risk factors as a baseline for the implementation of future education programs. Such programs can be especially effective where no screening or prevention measures exist (e.g., HPV vaccine) as in Palestine.

\section{Knowledge level of CC risk factors and its associated factors}

Good awareness of $\mathrm{CC}$, early detection and treatment remain the cornerstones to improve $\mathrm{CC}$ survival outcomes especially in low- and middle-income countries $[10,12,37,39]$. Only $23.7 \%$ of participants in this study 
Table 6 Multivariable logistic regression analyizing the association between the recognition of other risk factors and sociodemographic factors

\begin{tabular}{|c|c|c|c|c|c|c|c|c|c|}
\hline \multirow[t]{2}{*}{ Characteristic } & \multicolumn{3}{|c|}{$\begin{array}{l}\text { Not going for regular smear (pap) } \\
\text { tests }(n=3543)\end{array}$} & \multicolumn{3}{|c|}{$\begin{array}{l}\text { Having a husband who is not } \\
\text { circumcised }(n=2818)\end{array}$} & \multicolumn{3}{|c|}{$\begin{array}{l}\text { Having a husband with many } \\
\text { previous partners }(n=2562)\end{array}$} \\
\hline & \multirow[t]{2}{*}{ n (\%) } & \multirow[t]{2}{*}{$\operatorname{AOR}(95 \% \mathrm{Cl})^{\mathrm{a}}$} & \multirow[t]{2}{*}{$p$ value } & \multirow[t]{2}{*}{ n (\%) } & AOR $(95 \% \mathrm{Cl})^{\mathrm{a}}$ & \multirow[t]{2}{*}{$p$ value } & \multirow[t]{2}{*}{$\mathrm{n}(\%)$} & \multirow[t]{2}{*}{$\operatorname{AOR}(95 \% \mathrm{CI})^{\mathrm{a}}$} & \multirow[t]{2}{*}{$p$ value } \\
\hline \multicolumn{2}{|l|}{ Age group } & & & & & & & & \\
\hline $18-20$ & $407(11.5)$ & Ref & Ref & $346(12.3)$ & Ref & Ref & $266(10.4)$ & Ref & Ref \\
\hline $21-40$ & $2188(61.8)$ & $0.75(0.61-0.91)$ & 0.004 & $1752(62.2)$ & $0.88(0.72-1.07)$ & 0.19 & $1524(59.5)$ & $1.02(0.83-1.25)$ & 0.86 \\
\hline 41 or older & 948 (26.8) & $0.72(0.57-0.90)$ & 0.005 & 720 (25.6) & $0.79(0.63-0.99)$ & 0.043 & 772 (30.1) & $1.04(0.82-1.32)$ & 0.73 \\
\hline \multicolumn{10}{|l|}{ Educational level } \\
\hline Illiterate & $64(1.8)$ & Ref & Ref & $55(2.0)$ & Ref & Ref & 64 (2.5) & Ref & Ref \\
\hline Primary & $201(5.7)$ & $0.87(0.58-1.31)$ & 0.50 & $157(5.6)$ & $0.84(0.56-1.26)$ & 0.40 & $192(7.5)$ & $0.85(0.56-1.27)$ & 0.42 \\
\hline Preparatory & $569(16.1)$ & $0.98(0.67-1.44)$ & 0.94 & $409(14.5)$ & $0.80(0.55-1.17)$ & 0.26 & $433(16.9)$ & $0.69(0.47-1.01)$ & 0.06 \\
\hline Secondary & $1137(32.1)$ & $0.82(0.56-1.19)$ & 0.29 & $913(32.4)$ & $0.79(0.54-1.15)$ & 0.23 & $801(31.3)$ & $0.57(0.39-0.83)$ & 0.003 \\
\hline Diploma & $353(10.0)$ & $0.80(0.54-1.18)$ & 0.26 & $270(9.6)$ & $0.71(0.47-1.05)$ & 0.09 & $259(10.1)$ & $0.56(0.38-0.83)$ & 0.004 \\
\hline Bachelor & $1153(32.5)$ & $1.00(0.68-1.47)$ & 0.99 & $956(33.9)$ & $0.90(0.62-1.33)$ & 0.61 & 759 (29.6) & $0.56(0.38-0.83)$ & 0.003 \\
\hline Postgraduate & $66(1.9)$ & $0.97(0.58-1.63)$ & 0.92 & $58(2.1)$ & $1.01(0.60-1.68)$ & 0.98 & $54(2.1)$ & $0.77(0.46-1.28)$ & 0.31 \\
\hline \multicolumn{10}{|l|}{ Occupation } \\
\hline Unemployed/Housewife & $2414(68.1)$ & Ref & Ref & $1843(65.4)$ & Ref & Ref & $1734(67.7)$ & Ref & Ref \\
\hline Employed & $674(19.0)$ & $0.87(0.75-0.99)$ & 0.047 & $545(19.3)$ & $0.89(0.77-1.03)$ & 0.11 & $486(19.0)$ & $0.87(0.75-1.01)$ & 0.06 \\
\hline Retired & $22(0.6)$ & $0.62(0.36-1.06)$ & 0.08 & $19(0.7)$ & $0.77(0.44-1.34)$ & 0.35 & $29(1.1)$ & $1.24(0.74-2.06)$ & 0.41 \\
\hline Student & $433(12.2)$ & $0.85(0.69-1.06)$ & 0.15 & $411(14.6)$ & $1.32(1.07-1.63)$ & 0.011 & $313(12.2)$ & $1.19(0.96-1.49)$ & 0.12 \\
\hline \multicolumn{10}{|l|}{ Monthly income } \\
\hline$<1450$ NIS & $1369(38.6)$ & Ref & Ref & $1007(35.7)$ & Ref & Ref & 834 (32.6) & Ref & Ref \\
\hline$\geq 1450 \mathrm{NIS}$ & $2174(61.4)$ & $0.80(0.70-0.92)$ & 0.002 & $1811(64.3)$ & $0.92(0.80-1.05)$ & 0.23 & $1728(67.4)$ & $0.84(0.73-0.97)$ & 0.021 \\
\hline \multicolumn{10}{|l|}{ Residency } \\
\hline Gaza Strip & $1507(42.5)$ & Ref & Ref & $1127(40.0)$ & Ref & Ref & $842(32.9)$ & Ref & Ref \\
\hline WBJ & $2036(57.5)$ & $0.79(0.69-0.90)$ & 0.001 & $1691(60.0)$ & $0.89(0.78-1.02)$ & 0.09 & $1720(67.1)$ & $1.50(1.30-1.72)$ & $<0.001$ \\
\hline \multicolumn{10}{|l|}{ Having a chronic disease } \\
\hline No & $2871(81.0)$ & Ref & Ref & $2313(82.1)$ & Ref & Ref & $2011(78.5)$ & Ref & Ref \\
\hline Yes & $672(19.0)$ & $0.91(0.80-1.05)$ & 0.19 & 505 (17.9) & $0.88(0.77-1.01)$ & 0.07 & $551(21.5)$ & $1.01(0.88-1.16)$ & 0.87 \\
\hline \multicolumn{10}{|c|}{ Knowing someone with cancer } \\
\hline No & $1369(38.6)$ & Ref & Ref & $1150(40.8)$ & Ref & Ref & 1039 (40.6) & Ref & Ref \\
\hline Yes & $2174(61.4)$ & $1.40(1.27-1.55)$ & $<0.001$ & $1668(59.2)$ & $1.12(1.02-1.24)$ & 0.022 & $1523(59.4)$ & $1.09(0.98-1.20)$ & 0.12 \\
\hline \multicolumn{10}{|l|}{ Marital status } \\
\hline Single & $811(22.9)$ & Ref & Ref & $668(23.7)$ & Ref & Ref & $552(21.5)$ & Ref & Ref \\
\hline Married & $2557(72.2)$ & $0.97(0.84-1.13)$ & 0.70 & $2007(71.2)$ & $1.23(1.06-1.43)$ & 0.007 & $1880(73.4)$ & $1.20(1.03-1.41)$ & 0.019 \\
\hline Divorced & $82(2.3)$ & $1.24(0.87-1.75)$ & 0.23 & $65(2.3)$ & $1.37(0.97-1.94)$ & 0.07 & $58(2.3)$ & $1.22(0.86-1.74)$ & 0.27 \\
\hline Widowed & $93(2.6)$ & $1.00(0.71-1.40)$ & 0.99 & $78(2.8)$ & $1.43(1.02-2.01)$ & 0.039 & $72(2.8)$ & $1.00(0.71-1.42)$ & 0.99 \\
\hline Site of data collection & & & & & & & & & \\
\hline Public spaces & $1266(35.7)$ & Ref & Ref & $1147(40.7)$ & Ref & Ref & $976(38.1)$ & Ref & Ref \\
\hline Hospitals & $858(24.2)$ & $0.95(0.84-1.08)$ & 0.45 & $722(25.6)$ & $0.88(0.78-1.01)$ & 0.06 & $792(30.9)$ & $1.18(1.03-1.34)$ & 0.014 \\
\hline Primary healthcare centers & $1419(40.1)$ & $1.47(1.31-1.66)$ & $<0.001$ & $949(33.7)$ & $0.85(0.75-0.96)$ & 0.007 & $794(31.0)$ & $0.80(0.71-0.91)$ & 0.001 \\
\hline Characteristic & $\begin{array}{l}\text { ng married at } \\
=2197)\end{array}$ & $t$ a young age $(b e$ & efore age & & $\begin{array}{l}\text { Havir } \\
(\mathrm{n}=\end{array}$ & $\begin{array}{l}\text { ng many } \\
\text { 1597) }\end{array}$ & ildren (five & or more) & \\
\hline$n(0$ & & AOR $(95 \%$ & $\mathrm{Cl})^{\mathrm{a}}$ & $p$ value & $n(\%)$ & & AOR & $(95 \% \mathrm{Cl})^{\mathrm{a}}$ & $p$ value \\
\hline Age group & & & & & & & & & \\
\hline 18-20 & $1(11.0)$ & Ref & & Ref & 142 & (8.9) & Ref & & Ref \\
\hline $21-40$ & $8(60.0)$ & $1.03(0.83-1$ & 1.28) & 0.77 & 970 & (60.7) & 1.21 & $(0.95-1.55)$ & 0.12 \\
\hline 41 or older & $8(29.0)$ & $1.22(0.96-1$ & 1.56) & 0.10 & 485 & (30.4) & 1.47 & (1.11-1.94) & 0.007 \\
\hline
\end{tabular}


Table 6 (continued)

\begin{tabular}{|c|c|c|c|c|c|c|}
\hline \multirow[t]{2}{*}{ Characteristic } & \multicolumn{3}{|c|}{$\begin{array}{l}\text { Being married at a young age (before age 17) } \\
(n=2197)\end{array}$} & \multicolumn{3}{|c|}{$\begin{array}{l}\text { Having many children (five or more) } \\
(n=1597)\end{array}$} \\
\hline & n (\%) & $\operatorname{AOR}(95 \% \mathrm{Cl})^{\mathrm{a}}$ & $p$ value & n (\%) & $\operatorname{AOR}(95 \% \mathrm{Cl})^{\mathrm{a}}$ & $p$ value \\
\hline \multicolumn{7}{|l|}{ Educational level } \\
\hline Illiterate & $46(2.1)$ & Ref & Ref & $33(2.1)$ & Ref & Ref \\
\hline Primary & $115(5.2)$ & $0.73(0.48-1.12)$ & 0.15 & $93(5.8)$ & $0.91(0.57-1.45)$ & 0.70 \\
\hline Preparatory & $261(11.9)$ & $0.65(0.44-0.97)$ & 0.035 & $210(13.1)$ & $0.82(0.53-1.28)$ & 0.39 \\
\hline Secondary & $646(29.4)$ & $0.82(0.56-1.21)$ & 0.32 & $470(29.4)$ & $0.93(0.61-1.43)$ & 0.76 \\
\hline Diploma & $246(11.2)$ & $0.96(0.63-1.45)$ & 0.84 & $176(11.0)$ & $1.00(0.64-1.58)$ & 0.99 \\
\hline Bachelor & $821(37.4)$ & $1.19(0.80-1.77)$ & 0.39 & $571(35.8)$ & $1.22(0.79-1.89)$ & 0.37 \\
\hline Postgraduate & $62(2.8)$ & $1.67(0.99-2.82)$ & 0.054 & $44(2.8)$ & $1.59(0.90-2.81)$ & 0.11 \\
\hline \multicolumn{7}{|l|}{ Occupation } \\
\hline $\begin{array}{l}\text { Unemployed/house- } \\
\text { wife }\end{array}$ & $1345(61.2)$ & Ref & Ref & $1007(63.1)$ & Ref & Ref \\
\hline Employed & $517(23.5)$ & $0.99(0.85-1.15)$ & 0.86 & $389(24.4)$ & $1.04(0.88-1.22)$ & 0.66 \\
\hline Retired & $32(1.5)$ & $1.39(0.84-2.31)$ & 0.20 & $22(1.4)$ & $1.12(0.65-1.94)$ & 0.67 \\
\hline Student & $303(13.8)$ & $1.08(0.86-1.35)$ & 0.49 & $179(11.2)$ & $0.88(0.68-1.13)$ & 0.31 \\
\hline \multicolumn{7}{|l|}{ Monthly income } \\
\hline$<1450$ NIS & 717 (32.6) & Ref & Ref & $544(34.1)$ & Ref & Ref \\
\hline$\geq 1450$ NIS & $1480(67.4)$ & $0.85(0.73-0.99)$ & 0.032 & $1053(65.9)$ & $0.77(0.65-0.90)$ & 0.002 \\
\hline \multicolumn{7}{|l|}{ Residency } \\
\hline Gaza Strip & $779(35.5)$ & Ref & Ref & $559(35.0)$ & Ref & Ref \\
\hline WBJ & $1418(64.5)$ & $1.19(1.03-1.37)$ & 0.017 & $1038(65.0)$ & $1.30(1.11-1.52)$ & 0.001 \\
\hline \multicolumn{7}{|c|}{ Having a chronic disease } \\
\hline No & 1742(79.3) & Ref & Ref & $1268(79.4)$ & Ref & Ref \\
\hline Yes & $455(20.7)$ & $1.11(0.96-1.28)$ & 0.15 & $329(20.6)$ & $1.00(0.86-1.18)$ & 0.96 \\
\hline \multicolumn{7}{|c|}{ Knowing someone with cancer } \\
\hline No & $920(41.9)$ & Ref & Ref & $710(44.5)$ & Ref & Ref \\
\hline Yes & $1277(58.1)$ & $1.03(0.93-1.15)$ & 0.57 & $887(55.5)$ & $0.91(0.81-1.02)$ & 0.09 \\
\hline \multicolumn{7}{|l|}{ Marital status } \\
\hline Single & $579(26.4)$ & Ref & Ref & $396(24.8)$ & Ref & Ref \\
\hline Married & $1497(68.1)$ & $0.92(0.79-1.08)$ & 0.30 & $1099(68.8)$ & $0.85(0.72-1.01)$ & 0.07 \\
\hline Divorced & $58(2.6)$ & $1.17(0.82-1.66)$ & 0.39 & $51(3.2)$ & $1.36(0.94-1.96)$ & 0.10 \\
\hline Widowed & $63(2.9)$ & $0.96(0.67-1.36)$ & 0.81 & $51(3.2)$ & $0.99(0.68-1.45)$ & 0.98 \\
\hline \multicolumn{7}{|l|}{ Site of data collection } \\
\hline Public spaces & $921(41.9)$ & Ref & Ref & $631(39.5)$ & Ref & Ref \\
\hline Hospitals & $577(26.3)$ & $0.95(0.83-1.09)$ & 0.44 & $442(27.7)$ & $1.05(0.91-1.22)$ & 0.49 \\
\hline $\begin{array}{l}\text { Primary healthcare } \\
\text { centers }\end{array}$ & $699(31.8)$ & $0.85(0.75-0.97)$ & 0.013 & $524(32.8)$ & $0.93(0.81-1.07)$ & 0.34 \\
\hline
\end{tabular}

n, number of participants; $\mathrm{AOR}$, adjusted odds ratio; $\mathrm{Cl}$, confidence interval; WBJ, West Bank and Jerusalem

${ }^{\text {a }}$ Adjusted for age-group, educational level, occupation, monthly income, marital status, residency, having a chronic disease, knowing someone with cancer, and site of data collection

had a good level of knowledge of CC risk factors, which is similar to reports from Tunisia, Libya, Qatar, and Oman [34, 35, 40, 41]. The relatively lower incidence and mortality rates in these Arab countries might have driven the health authorities to focus on educating women about other types of cancers that have higher rates (e.g., breast cancer) [42]. Education campaigns can be costly, and their funding is usually limited. However, the long-term investment in raising public awareness of CC risk factors may lead to prevention and early diagnosis of $\mathrm{CC}$ reducing the financial burden associated with treatment.

Low and colleagues reported better knowledge of CC risk factors among British women who knew someone with cancer, in concordance with this study and other studies in the United Kingdom [43, 44]. A possible explanation could be that women who know someone with 
cancer are expected to take care and accompany them during healthcare visits. Therefore, these women may come across more experience and knowledge about health-related topics. Furthermore, women's concerns about someone's health might lead them to read more about their diagnosis.

Married women were more likely than single women to recognize most of the $\mathrm{CC}$ risk factors in this study, which is in concordance with results of other studies [34, 35, 40]. Married women are expected to be more knowledgeable of topics related to reproductive and sexual health through their visits to healthcare facilities and, thus, also have higher chances to access more accurate information from healthcare professionals. In addition, married women may educate themselves by reading printed health materials distributed in clinics or by using internet resources. On the other hand, single women in conservative communities, such as Palestine, may feel inhibited to read or talk about sexual and reproductive health issues. In fact, the data collectors noticed this when they asked single women about risk factors related to sex, such as having a husband with many previous partners, marrying a husband who is not circumcised, and infection with an STI. Furthermore, although some health-related topics are part of the school curriculum in Palestine, topics around sexual health and CC are not included, potentially increasing the barriers of single women to address these topics. Therefore, education interventions should be tailored to address the emotional barriers of single women to promote their willingness to know more about reproductive health topics including CC.

Low socioeconomic status is one of the CC risk factors, raising the importance of improving the awareness of these factors in this group of women for prevention and early detection of CC [45]. In this study, being employed and having a high monthly income were associated with a decrease in the odds of having good knowledge. The decrease in the likelihood of having good knowledge associated with higher monthly income might only reflect the fact that women in the Gaza Strip know more than those in the WBJ but have less income. Furthermore, employed women were less likely to have a good knowledge, which might reflect that more single women might be employed than married women. In addition, those employed women might have less time to read about health-related topics and less involvement in social interactions where women talk about their own and their relatives' experiences including those health issues. Another contributing factor might be that unemployment is higher in the Gaza Strip compared with the WBJ and this includes women, so that this might be another reflection of the generally better knowledge amongst women from Gaza compared with those from WBJ [46]. In contrast to this, previous studies showed that employed women and those having a high monthly income were more likely to have a good knowledge level of CC risk factors [35, 40, 47].

Higher education level was shown to be associated with more uptake of CC prevention and early detection strategies $[48,49]$. Similar to previous studies on cancer awareness in the Gaza Strip, participants with only a secondary or diploma degree in this study showed lower likelihood to have good knowledge of CC risk factors [28-30]; highlighting the lack of such topics within the Palestinian school curricula. There is a need to revise school curricula to include a wider range of health-related topics. Kyle and colleagues demonstrated that a school-based educational intervention improved the recall and recognition of most of the cancer signs and symptoms even after six months from the intervention [50]. Raising such awareness among adolescents could be useful as this might shape their health-related behaviors in the future.

\section{Recognition of CC risk factors in the Gaza strip versus the WBJ}

The participants from the Gaza Strip were more likely than participants from the WBJ to recognize 8 out of 11 $\mathrm{CC}$ risk factors. A possible contributing factor could be that living in extended families is more notable in the Gaza Strip. This could increase the likelihood of sharing and discussing health-related experiences or relatives' stories, which may help in shaping women's knowledge. Another form of interaction that could play a role in building women's knowledge is the interaction with healthcare professionals. Women in the WBJ encounter several challenges in accessing healthcare facilities due to the Israeli checkpoints between geographical areas. These checkpoints restrict their movement and impede access to healthcare services [51, 52]. In contrast to this, movement within the Gaza Strip is easy and unrestricted for women, so that most women in the Gaza Strip can access healthcare facilities easily and shape their knowledge while communicating with healthcare providers $[53,54]$. Moreover, women in the Gaza Strip have a relatively higher fertility rate among women of childbearing age (15-49 years) compared with those in the WBJ (4.5 vs 3.7 births per woman), therefore, they may be exposed to more experience in sexual and reproductive healthcare and associated health education [55].

\section{Recognizing $\mathrm{CC}$ risk factors}

In this study, women recognized 'infection with an STI' more than 'infection with HPV'. This is similar to findings among Libyan and British women [35, 43], which suggests that women are more aware of the link between $\mathrm{CC}$ and STIs than causative micro-organisms (e.g., HPV) 
as reported in the literature $[35,43,56]$. Future educational campaigns should highlight the role of HPV in CC etiology.

Having five or more children was the least risk factor reported in this study. This is similar to findings of other studies conducted in Libya, United States, India, Oman, and Malaysia [35, 47, 57-59]. A possible explanation for this could be that women's thoughts of CC risk factors are shaped by the culture of the country where they were raised. Palestinian culture encourages having many children as a source of kinship and wealth. Therefore, this might have prevented Palestinian women to consider negative associations with multiparty, such as it being a risk factor of CC. Moreover, more than $70.0 \%$ of study participants were married and, considering the high fertility rate in Palestine, which might also have had contributed to shaping such beliefs about multiparity [55]. Education interventions should focus on 'having many children' as a risk factor of CC since this is very relevant to the Palestinian society.

\section{Future directions}

The findings of this study reflect the need to promote educational programs to improve women's knowledge of $\mathrm{CC}$ in Palestine. Enriching school curricula with healthrelated topics and targeting women in the reproductive age should be prioritized. This could drive these women to adjust their behavioral risk factors, hence, decrease their chance of developing CC. In addition, raising young women's awareness of CC may make them more confident to talk about any possible CC symptom and less embarrassed to seek medical advice or discuss their concerns with healthcare professionals.

\section{Strengths and limitations}

The main strengths of this study included the large sample size and the high response rate. In addition, the stratified approach that may provide a representative view of the target population's knowledge on different levels of the Palestinian community.

Limitations of this study included the convenience sampling that may limit the generalizability of the findings. However, this may be alleviated by the recruiting a large number of participants while having a high response rate and covering different geographical areas in Palestine. Another limitation could be the exclusion of visitors or patients in the oncology departments and participants with medical backgrounds, possibly decreasing the number of participants with a presumably good level of knowledge. On the other hand, their exclusion was meant to increase the relevancy of this study as a measure of knowledge among the public.

\section{Conclusion}

The overall knowledge of women about CC risk factors was low with only $23.7 \%$ of participants demonstrating good knowledge of $\mathrm{CC}$ risk factors. Knowing someone with cancer was the only factor associated with an increase in the odds of having good knowledge. Conversely, completing only secondary or diploma degree, being employed, and having a monthly income of $\geq 1450$ NIS were all associated with a decrease in the odds of having good knowledge. Introducing topics around sexual and reproductive health, including CC risk factors and symptoms, in school curricula as well as public discourse could be one way of bridging this gap.

\section{Abbreviations}

CC: Cervical cancer; HPV: Human papillomavirus; WBJ: West Bank and Jerusalem; PHCs: Primary healthcare centers; MoH: Ministry of health; CeCAM: Cervical cancer awareness measure; Cl: Confidence interval; OR: Odds ratio; STI: Sexually transmitted infection.

\section{Supplementary Information}

The online version contains supplementary material available at https://doi. org/10.1186/s12905-021-01510-2.

Additional file 1. Results of all bivariable logistic regression analyses.

\section{Acknowledgements}

The authors thank all the participants for their time to be part of our study.

\section{Authors' contributions}

$M E, M T$, and $H A$ contributed to design of the study, data analysis, data interpretation, and drafting of the manuscript. IA, MA, AR, AA, SK, LK, NF, BA, LiK, HK, DE, NA, AN, TA, ZA, SI, GT, MH, HAS, ZAH, HH, MZ, RS, LH, SR, HaA, TR, RZ, and AmA contributed to design of the study, data collection, data entry, and data interpretation. NAE and BB contributed to design of the study, data interpretation, drafting of the manuscript, and supervision of the work. All authors have read and approved the final manuscript. Each author has participated sufficiently in the work to take public responsibility for the content. All authors read and approved the final manuscript.

\section{Availability of data and materials}

The dataset used and analyzed during the current study is available from the corresponding author upon reasonable request.

\section{Declarations}

\section{Ethics approval and consent to participate}

The study was approved by the Helsinki Committee in the Gaza Strip, a committee within the $\mathrm{MoH}$ that gives study approvals. In addition, ethical approval was also obtained from the Islamic University of Gaza Research Ethics Committee. An approval to conduct the study at the governmental hospitals and PHCs was also obtained from the Human Resources Development department at the Palestinian $\mathrm{MoH}$. All the methods of the study were carried out in accordance with relevant local guidelines and regulations. Informed consents were obtained from all participants after giving a detailed explanation of the study and its purpose. All participants were informed that their participation was completely voluntary and that their decision would not affect the medical care they receive. Data confidentiality was maintained throughout the study.

Consent for publication

Not applicable. 


\section{Competing interests}

All authors declare no competing interests.

\section{Author details}

${ }^{1}$ Harvard Medical School, 25 Shattuck Street, Boston, MA 02115, USA. ${ }^{2}$ Ministry of Health, Gaza, Palestine. ${ }^{3}$ Faculty of Medicine, Al-Quds University, Jerusalem, Palestine. ${ }^{4}$ Faculty of Medicine, Islamic University of Gaza, Gaza, Palestine. ${ }^{5}$ Almakassed Hospital, Jerusalem, Palestine. ${ }^{6}$ Faculty of Graduate Studies, An-Najah National University, Nablus, Palestine. ${ }^{7}$ Faculty of Medicine, An-Najah National University, Nablus, Palestine. ${ }^{8}$ Faculty of Pharmacy, Alazhar University of Gaza, Gaza, Palestine. ${ }^{9}$ Faculty of Dentistry and Dental Surgery, Al-Quds University, Jerusalem, Palestine. ${ }^{10}$ Faculty of Medicine, Alazhar University of Gaza, Gaza, Palestine. ${ }^{11}$ Alia Hospital, Hebron, Palestine. ${ }^{12}$ Al-Shiffa Hospital, Gaza, Palestine. ${ }^{13}$ Faculty of Nursing, Islamic University of Gaza, Gaza, Palestine.

Received: 16 April 2021 Accepted: 12 October 2021

Published online: 02 November 2021

\section{References}

1. Sung H, Ferlay J, Siegel RL, Laversanne M, Soerjomataram I, Jemal A, et al. Global cancer statistics 2020: GLOBOCAN estimates of incidence and mortality worldwide for 36 cancers in 185 countries. CA Cancer J Clin. 2021;71(3):209-49.

2. World Health Organization. Improving data for decision-making: a toolkit for cervical cancer prevention and control programmes. https://bit.ly/ 2RhPwAr. Accessed 7 Apr 2021.

3. International Agency for Research on Cancer. GLOBOCAN 2020: estimated cancer incidence, mortality and prevalence Gaza strip and west bank in 2020. https://bit.ly/2QUyO9Y. Accessed 7 Apr 2021

4. The World Bank. Data for lower middle income. https://bit.ly/31N7Cw5. Accessed 7 Apr 2021.

5. Arbyn M, Weiderpass E, Bruni L, De Sanjosé S, Saraiya M, Ferlay J, et al. Estimates of incidence and mortality of cervical cancer in 2018: a worldwide analysis. Lancet Glob Health. 2020;8(2):e191-203.

6. Ott JJ, Ullrich A, Miller AB. The importance of early symptom recognition in the context of early detection and cancer survival. Eur J Cancer. 2009;45(16):2743-8.

7. McPhail S, Johnson S, Greenberg D, Peake M, Rous B. Stage at diagnosis and early mortality from cancer in England. Br J Cancer. 2015:112(S1):S108-15.

8. Hull R, Mbele M, Makhafola T, Hicks C, Wang SM, Reis R, et al. Cervical cancer in low and middle-income countries (review). Oncol Lett. 2020;20(3):2058-74

9. Simon AE, Wardle J, Grimmett C, Power E, Corker E, Menon U, et al. Ovarian and cervical cancer awareness: development of two validated measurement tools. J Family Plan Reprod Health Care. 2012;38(3):167-74.

10. World Health Organization. Comprehensive cervical cancer control: a guide to essential practice, 2nd edition. https://bit.ly/31QUiqy. Accessed 7 Apr 2021.

11. World Heatlh Organization. WHO director-general calls for all countries to take action to help end the suffering caused by cervical cancer. https:// bit.ly/3uvj2AV. Accessed 7 Apr 2021.

12. World Health Organization. Human papillomavirus (HPV) and cervical cancer. https://bit.ly/3dLD3fl. Accessed 7 Apr 2021.

13. Wardak S. Human papillomavirus (HPV) and cervical cancer. Med Dosw Mikrobiol. 2016;68(1):73-84.

14. Okunade KS. Human papillomavirus and cervical cancer. J Obstet Gynaecol. 2020;40(5):602-8.

15. de Martel C, Plummer M, Vignat J, Franceschi S. Worldwide burden of cancer attributable to HPV by site, country and HPV type. Int J Cancer. 2017;141(4):664-70.

16. Arbyn M, Xu L, Simoens C, Martin-Hirsch PP. Prophylactic vaccination against human papillomaviruses to prevent cervical cancer and its precursors. Cochrane Database Syst Rev. 2018:5:CD009069.

17. Louie KS, De Sanjose S, Diaz M, Castellsagué X, Herrero R, Meijer CJ, et al Early age at first sexual intercourse and early pregnancy are risk factors for cervical cancer in developing countries. Br J Cancer. 2009;100(7):1191-7.

18. Morris BJ, Hankins CA, Banerjee J, Lumbers ER, Mindel A, Klausner JD, et al. Does male circumcision reduce women's risk of sexually transmitted infections, cervical cancer, and associated conditions? Front Public Health. 2019;7:4.

19. Sugawara Y, Tsuji I, Mizoue T, Inoue M, Sawada N, Matsuo K, et al. Cigarette smoking and cervical cancer risk: an evaluation based on a systematic review and meta-analysis among Japanese women. Jpn J Clin Oncol. 2019;49(1):77-86.

20. Kashyap N, Krishnan N, Kaur S, Ghai S. Risk factors of cervical cancer: a case-control study. Asian Pac J Oncol Nurs. 2019;6(3):308-14.

21. Harper DM, Demars LR. Primary strategies for HPV infection and cervical cancer prevention. Clin Obstet Gynecol. 2014;57(2):256-78.

22. Kumar RV, Bhasker S. Potential opportunities to reduce cervical cancer by addressing risk factors other than HPV. J Gynecol Oncol. 2013;24(4):295.

23. Whitaker KL, Smith CF, Winstanley K, Wardle J. What prompts help-seeking for cancer 'alarm' symptoms? A primary care based survey. $\mathrm{Br} J$ Cancer. 2016;114(3):334-9.

24. Simon AE, Waller J, Robb K, Wardle J. Patient Delay in presentation of possible cancer symptoms: the contribution of knowledge and attitudes in a population sample from the United Kingdom. Cancer Epidemiol Biomark Prev. 2010;19(9):2272-7.

25. Esteva M, Leiva A, Ramos M, Pita-Fernández S, González-Luján L, Casamitjana $M$, et al. Factors related with symptom duration until diagnosis and treatment of symptomatic colorectal cancer. BMC Cancer. 2013;13(1):87.

26. Palestinian Ministry of Health. Annual report for ministry of health in Palestine 2019. https://bit.ly/39FtEFR. Accessed 4 Apr 2021.

27. World Health Organization. Research Tools: Process of translation and adaptation of instruments. https://bit.ly/327BfbT. Accessed 7 Apr 2021.

28. Elshami M, Elshami A, Alshorbassi N, Alkhatib M, Ismail I, Abu-Nemer K, et al. Knowledge level of cancer symptoms and risk factors in the Gaza Strip: a cross-sectional study. BMC Public Health. 2020;20(1):1-11.

29. Elshami M, Abu Kmeil H, Abu-Jazar M, Mahfouz I, Ashour D, Aljamal A, et al. Breast cancer awareness and barriers to early presentation in the gaza-strip: a cross-sectional study. J Global Oncol. 2018;4:1-13.

30. Elshami M, Alfaqawi M, Abdalghafoor T, Nemer AA, Ghuneim M, Lubbad $\mathrm{H}$, et al. Public awareness and barriers to seeking medical advice for colorectal cancer in the gaza strip: a cross-sectional study. J Global Oncol. 2019(5):JGO.18.00252.

31. Elshami M, Bottcher B, Alkhatib M, et al. Perceived barriers to seeking cancer care in the Gaza Strip: a cross-sectional study. BMC Health Serv Res. 2021;21:28.

32. Harvard Humanitarian Initiative. KoBoToolbox. https://www.kobotoolbox org. Accessed 7 Apr 2021.

33. Palestinian Central Bureau of Statistics. On the occasion of the International Workers' Day, Dr. Awad, presents the current status of the Palestinian labour force. https://bit.ly/3diTdgy. Accessed 7 Apr 2021.

34. El Mhamdi S, Bouanene I, Mhirsi A, Bouden W, Soussi SM. Cervical cancer screening: women's knowledge, attitudes, and practices in the region of Monastir (Tunisia). Rev Epidemiol Sante Publique. 2012;60(6):431-6.

35. Hweissa NA, Su TT. Awareness of cervical cancer and socio-demographic variations among women in Libya: an exploratory study in Az-Zawiya city. Eur J Cancer Care. 2018;27(1):e12750.

36. Narayana G, Suchitra MJ, Sunanda G, Ramaiah JD, Kumar BP, Veerabhadrappa KV. Knowledge, attitude, and practice toward cervical cancer among women attending obstetrics and gynecology department: a cross-sectional, hospital-based survey in South India. Indian J Cancer. 2017:54(2):481-7.

37. World Health Organization. Guide to cancer early diagnosis 2017. https:// bit.ly/3fNYxeV. Accessed 4 Apr 2021

38. Asl RT, Osch LV, Vries ND, Zendehdel K, Shams M, Zarei F, et al. The role of knowledge, risk perceptions and cues to action among Iranian women concerning cervical cancer and screening: a qualitative exploration. BMC Public Health. 2020;20:1-2.

39. Randall TC, Ghebre R. Challenges in prevention and care delivery for women with cervical cancer in Sub-Saharan Africa. Front Oncol. 2016:6:160.

40. Al-Meer FM, Aseel MT, Al-Khalaf J, Al-Kuwari MG, Ismail MFS. 855 Knowledge, attitude and practices regarding cervical cancer and screening among women visiting primary health care in Qatar. East Mediterr Health J. 2011;17(11):855-61.

41. Nasar A, Waad A, Atheer A, Nasra A. Awareness of cervical cancer and pap smear testing among Omani women. Asian Pac J Cancer Prev. 2016:17(11):4825-30. 
42. International Agency for Research on Cancer. Cervix Uteri Fact Sheet. https://bit.ly/3cz4lan. Accessed 4 Apr 2021.

43. Low EL, Simon AE, Lyons J, Romney-Alexander D, Waller J. What do British women know about cervical cancer symptoms and risk factors? Eur J Cancer. 2012;48(16):3001-8.

44. Adlard J, Hume M. Cancer knowledge of the general public in the United Kingdom: survey in a primary care setting and review of the literature. Clin Oncol (R Coll Radiol (Great Br)). 2003;15 4:174-80.

45. American Cancer Society. Risk factors for cervical cancer. https://bit.ly/ 2S2y4QU. Accessed 17 May 2021.

46. Palestinian Central Bureau of Statistics. Labour force survey (OctoberDecember, 2018) round (Q4/2018). https://bit.ly/3yvpJWq. Accessed 21 May 2021.

47. Ralston JD, Taylor VM, Yasui Y, Kuniyuki A, Jackson JC, Tu S-P. Knowledge of cervical cancer risk factors among Chinese immigrants in Seattle. J Commun Health. 2003;28(1):41-57.

48. Murfin J, Irvine F, Meechan-Rogers R, Swift A. Education, income and occupation and their influence on the uptake of cervical cancer prevention strategies: a systematic review. J Clin Nurs. 2020;29(3-4):393-415.

49. Nene B, Jayant K, Arrossi S, Shastri S, Budukh A, Hingmire S, et al. Determinants of womens participation in cervical cancer screening trial, Maharashtra. India Bull World Health Organ. 2007;85(4):264-72.

50. Kyle RG, Forbat L, Rauchhaus P, Hubbard G. Increased cancer awareness among British adolescents after a school-based educational intervention: a controlled before-and-after study with 6-month follow-up. BMC Public Health. 2013;13(1):190.

51. Devi S. Health in the West Bank. The Lancet. 2007;370(9596):1405-6.
52. Health in the West Bank. United Nations Relief and Works Agency for Palestine Refugees in the Near East: https://bit.ly/3uoUj1 m. Accessed 7 Apr 2021.

53. Hamdan M, Defever M, Abdeen Z. Organizing health care within political turmoil: the Palestinian case. Int J Health Plan Manag. 2003;18(1):63-87.

54. Palestinian Central Bureau of Statistics | Dr. Ola Awad, presents a brief on the status of Palestinian people at the end of 2019. https://bit.ly/31P2cAE. Accessed 7 Apr 2021.

55. Palestinian Ministry of Health. Health annual report in Palestine 2016. https://bit.ly/3whx4Yv. Accessed 7 Apr 2021.

56. Moore AR, Driver N. Knowledge of cervical cancer risk factors among educated women in Lomé, Togo: half-truths and misconceptions. SAGE Open. 2014;4(4):215824401455704.

57. Singh S, Narayan N, Sinha R, Sinha P, Sinha VP, Upadhye JJ. Awareness about cervical cancer risk factors and symptoms. Int J Reprod Contracept Obstet Gynecol. 2018;7(12):4987.

58. Alwahaibi N, Alsalami W, Alramadhani N, Alzaabi A. Factors influencing knowledge and practice regarding cervical cancer and pap smear testing among Omani women. Asian Pac J Cancer Prev. 2018;19(12):3367-74.

59. Seng LM, Rosman AN, Khan A, Haris NM, Mustapha NAS, Husaini NSM, et al. Awareness of cervical cancer among women in Malaysia. Int J Health Sci (Qassim). 2018;12(4):42-8.

\section{Publisher's Note}

Springer Nature remains neutral with regard to jurisdictional claims in published maps and institutional affiliations.
Ready to submit your research? Choose BMC and benefit from:

- fast, convenient online submission

- thorough peer review by experienced researchers in your field

- rapid publication on acceptance

- support for research data, including large and complex data types

- gold Open Access which fosters wider collaboration and increased citations

- maximum visibility for your research: over 100M website views per year

At BMC, research is always in progress.

Learn more biomedcentral.com/submissions 